Pacific Journal of Mathematic 


\title{
INTEGRATION WITH RESPECT TO VECTOR MEASURES
}

\author{
D. R. LEWIS
}

The purpose of this paper is to develop a theory of integration with respect to measures into a locally convex, Hausdorff linear topological space, using a linear functional approach.

Section 1 presents some basic facts about such measures, chiefly through the study of their $p$-semi-variations (Definition 1.2). Devices of this sort have been considered by other authors (see [1], [4]), but chiefly to give expressions for the norms of linear operators defined by vector measures. We consider the continuity properties of the $p$ semi-variation, and define regularity in terms of the $p$-semi-variation.

The integration theory is developed in $\S 2$. Although the integral is defined in terms of linear functionals, it is in no sense a weak integral. The dominated (strong) convergence theorem is proven under the additional assumption that the limit function is integrable, and it is shown that this is true whenever the range space of the measure is sequentially complete.

In $\S 3$ integral representations of weakly compact operators from $C(S), C_{0}(T), C_{c}(T)$ and $C(T)_{\beta}$ into a locally convex, Hausdorff space are given. We used these representations to show that the above spaces satisfy a strengthened rersion of the Dunford-Pettis Propertyspecifically that a weakly compact operator on these spaces maps weakly Cauchy sequences into convergent sequences, without any assumption about the completeness of the range of the operator.

1. Throughout the first two sections $(S, \Sigma)$ denotes a measurable space, $(X, \mathscr{T})$ a complex, locally convex Hausdorff linear topological space with dual $X^{*}$ and $\mu$ an additive set function from $\Sigma$ into $X$. If $x^{*} \in X^{*}$ and $p$ is a semi-norm on $X$ we will write $x^{*} \leqq p$ whenever $\left|x^{*}(x)\right| \leqq p(x)$ for all $x \in X$. The following theorem, stated here without proof, was first proved by Pettis [6] for normed spaces and Grothendieck [5] for locally convex spaces.

THEOREM 1.1. If $\mu$ is countably additive in the weak topology, then $\mu$ is countably additive in $\mathscr{T}$.

Definition 1.2. If $p$ is a semi-norm on $X$, then the $p$-semivariation of $\mu$ is the function from $\Sigma$ into the extended reals defined by $\|\mu\|_{p}(E)=\sup _{x^{*} \leqq p} v\left(x^{*} \mu, E\right)$, where $v\left(x^{*} \mu, \cdot\right)$ is the scalar variation of 
$x^{*} \mu$.

It follows immediately that $\|\mu\|_{p}(\cdot)$ is monotone, subadditive and that $p[\mu(E)] \leqq\|\mu\|_{p}(E) \leqq 4 \sup _{F \subset E} p[\mu(F)]$ for each $E \in \Sigma$. If $\mu$ is a measure then $\|\mu\|_{p}(\cdot)$ is countably subadditive and real valued, since the range of $\mu$ is bounded.

THEOREM 1.3. If $\mu$ is a measure, $p$ a continuous semi-norm and $\left(E_{n}\right)$ a convergent sequence in $\Sigma$, then $\|\mu\|_{p}\left(\lim _{n} E_{n}\right)=\lim _{n}\|\mu\|_{p}\left(E_{n}\right)$.

Proof. We first establish a special case. Suppose $\left(E_{n}\right)$ is a decreasing sequence in $\Sigma$ with empty intersection and that, for some $\varepsilon>0,\|\mu\|_{p}\left(E_{n}\right)>\varepsilon$ for each $n$. Let $n_{1}=1$. For some $x^{*} \leqq p$ and $n_{2}>n_{1}, v\left(x^{*} \mu, E_{n_{1}}\right)>\varepsilon$ and $v\left(x^{*} \mu, E_{n_{2}}\right)<\varepsilon / 2$. Then also $4 \sup _{F \subset E n_{1} \backslash E n_{2}}$ $p[\mu(F)] \geqq v\left(x^{*} \mu, E_{n_{1}} \backslash E_{n_{2}}\right)>\varepsilon / 2$, so for some $F_{1} \subset E_{n_{1}} \backslash E_{n_{2}}$ we have

$$
p\left[\mu\left(F_{1}\right)\right]>\frac{\varepsilon}{8} .
$$

Continuing in this manner there is an increasing sequence $\left(n_{k}\right)$ of positive integers and a sequence $\left(F_{k}\right)$ in $\Sigma$ such that $F_{k} \subset E_{n_{k} \backslash} E_{n_{k+1}}$ and $p\left[\mu\left(F_{k}\right)\right]>\varepsilon / 8$ for each $k$. This contradicts the countable additivity of $\mu$ since the $F_{k}$ 's are pairwise disjoint.

If $\left(E_{n}\right)$ is in $\Sigma$ and has limit $E$, then $\|\mu\|_{p}(E)=\lim _{n}\|\mu\|_{p}\left(E_{n}\right)$ since the inequality

$$
\|\| \mu\left\|_{p}(E)-\right\| \mu\left\|_{p}\left(E_{n}\right) \mid \leqq\right\| \mu\left\|_{p}\left[\bigcup_{k \geqq n}\left(E \backslash E_{k}\right)\right]+\right\| \mu \|_{p}\left[\bigcup_{k \geqq n}\left(E_{k} \backslash E\right)\right]
$$

holds.

COROLlaRY 1.4. If $\mu$ is a measure and $\left(E_{n}\right)$ is a convergent sequence in $\Sigma$, then $\mu\left(\lim _{n} E_{n}\right)=\lim _{n} \mu\left(E_{n}\right)$.

Proof. Let $E=\lim _{n} E_{n}$. The corollary follows since

$$
\lim _{n}\left(E \backslash E_{n}\right)=\lim _{n}\left(E_{n} \backslash E\right)=\varnothing
$$

and $p\left[\mu(E)-\mu\left(E_{n}\right)\right]<\|\mu\|_{p}\left(E \backslash E_{n}\right)+\|\mu\|_{p}\left(E_{n} \backslash E\right)$ for each $n$ and semi-norm $p$.

Definition 1.5. Suppose $S$ is a topological space. $\mu$ is regular (in $\mathscr{T}$ ) if, for each $E \in \Sigma \varepsilon>0$ and continuous semi-norm $p$ on $X$, there is a relatively compact set $K$ in $\Sigma$ whose closure is contained in $E$ and a set $G$ in $\Sigma$ whose interior contains $E$ such that

$$
\|\mu\|_{p}(G \backslash K)<\varepsilon \text {. }
$$


If $\mu$ is bounded and regular, then $x^{*} \mu$ is bounded and regular for each $x^{*} \in X^{*}$. This implies that $x^{*} \mu$ is countably additive for each $x^{*} \in X^{*}$, which in turn implies that $\mu$ is a measure. Also, regularity in the weak topology is equivalent to the regularity of $x^{*} \mu$ for each $x^{*} \in X^{*}$.

THEOREM 1.6. If $\mu$ is regular in the weak topology and $\mu$ is a measure, then $\mu$ is regular in $\mathscr{T}$.

Proof. If $\mu$ is not inner regular there is an $E \in \Sigma$, a positive $\varepsilon$ and a $\mathscr{T}$-continuous semi-norm $p$ such that $\|\mu\|_{p}(E \backslash K)>\varepsilon$ for each relatively compact $K$ in $\Sigma$ with $\bar{K} \subset E$. Let $K_{1}=\varnothing$. There is an $x_{1} \leqq p$ and a relatively compact set $K_{2}$ in $\Sigma$ such that

$$
K_{1} \subset K_{2}, \bar{K}_{2} \subset E, v\left(x_{1} \mu, E \backslash K_{1}\right)>\varepsilon
$$

and $v\left(x_{1} \mu, E \backslash K_{2}\right)<\varepsilon / 2$. Since $\|\mu\|_{p}\left(E \backslash K_{2}\right)>\varepsilon$ we may continue this process and obtain a sequence $\left(x_{n}\right)$ of functionals dominated by $p$ and an increasing sequence $\left(K_{n}\right)$ of relatively compact sets in $\Sigma$ such that $\bar{K}_{n} \subset E, v\left(x_{n} \mu, E \backslash K_{n}\right)>\varepsilon$ and $v\left(x_{n} \mu, E \backslash K_{n+1}\right)<\varepsilon / 2$ for each $n$. Let $K=\mathbf{U}_{n \geqq 1} K_{n}$. Since $\lim _{n}\left(K \backslash K_{n}\right)=\varnothing$ there is an $m$ such that

$$
\|\mu\|_{p}\left(K \backslash K_{m}\right)<\frac{\varepsilon}{2} .
$$

But then $v\left(x_{m} \mu, E \backslash K_{m}\right) \leqq v\left(x_{m} \mu, E \backslash K_{m+1}\right)+\|\mu\|_{p}\left(K \backslash K_{m}\right)<\varepsilon . \quad$ The outer regularity of $\mu$ is proven similarly.

2. Throughout this section $\mu$ is a fixed measure from $\Sigma$ into $(X, \mathscr{T})$ and $\phi$ the complex numbers.

Definition 2.1. A function $f: S \rightarrow \phi$ is $\mu$-integrable if

(1) $f$ is $x^{*} \mu$-integrable for each $x^{*} \in X^{*}$, and

(2) for each $E \in \Sigma$ there is an element of $X$, denoted by

$$
\int_{E} f(t) \mu(d t)
$$

such that $x^{*} \int_{E} f(t) \mu(d t)=\int_{E} f(t) x^{*} \mu(d t)$ for each $x^{*} \in X^{*}$.

Since $(X, \mathscr{T})$ is Hausdorff the integral is well-defined. No assumption is made about the completeness of $(X, \mathscr{T})$. $\mathscr{T}$ enters into the definition of the integral only in that it determines $X^{*}$, so that in any of the results of this section $\mathscr{T}$ may be replaced by any topology in the Mackey spectrum of $(X, \mathscr{T})$.

It is easy to see that the integral has the following properties: 
(1) The integral is linear.

(2) Every simple function $\sum_{i \leqq n} a_{i} \chi_{E_{i}}$ is $\mu$-integrable and

$$
\int_{E}\left(\sum_{i \leqq n} a_{i} \chi_{E_{i}}\right)(t) \mu(d t)=\sum_{i \leqq n} a_{i} \mu\left(E \cap E_{i}\right)
$$

for $E \in \Sigma$.

(3) If $f$ is bounded and $\mu$-integrable, then

$$
p\left[\int_{E} f(t) \mu(d t)\right] \leqq\|\mu\|_{p}(E) \cdot \sup _{s \in S}|f(s)|
$$

for each $E \in \Sigma$ and continuous semi-norm $p$.

(4) If $f$ is $\mu$-integrable and $T$ is a continuous linear operator from $X$ into a locally convex Hausdorff space $Y$, then $f$ is $T \mu$-integrable and $\int_{E} f(t) T \mu(d t)=T \int_{E} f(t) \mu(d t)$ for $E \in \Sigma$.

THEOREM 2.2. (1) If $f$ is $\mu$-integrable, then the set function on $\Sigma$ defined by $\Phi(E)=\int_{E} f(t) \mu(d t)$ is a measure,

$$
\|\Phi\|_{p}(E)=\sup _{x^{*} \leqq p} \int_{E}|f(t)| v\left(x^{*} \mu, d t\right) \text { and } \lim _{\|\mu\|_{p}(E) \rightarrow 0}\|\Phi\|_{p}(E)=0
$$

for each continuous semi-norm $p$.

(2) Let $\left(f_{n}\right)$ be a sequence of $\mu$-integrable functions which converge pointwise to $f$ on $S$ and $g$ be a $\mu$-integrable function such that $\left|f_{n}\right| \leqq|g|$ for each $n$. $f$ is $\mu$-integrable if $X$ is sequentially complete. If $f$ is $\mu$-integrable then

$$
\int_{E} f(t) \mu(d t)=\lim _{n} \int_{E} f_{n}(t) \mu(d t)
$$

uniformly with respect to $E \in \Sigma$.

Proof. The set function $\Phi$ in (1) is a measure by Pettis's Theorem, and the expression for its $p$-semi-variation is correct since

$$
v\left(x^{*} \Phi, E\right)=\int_{E}|f(t)| v\left(x^{*} \mu, d t\right)
$$

for each $x^{*}$. Clearly $\|\Phi\|_{p}(E)=0$ whenever $\|\mu\|_{p}(E)=0$, so in light of Theorem 1.3 the usual proof by contradiction that zero-zero and $\varepsilon-\delta$ absolute continuity are equivalent in finite measure spaces establishes that $\lim _{\|\mu\|_{p}(E) \rightarrow 0}|| \Phi \|_{p}(E)=0$.

To prove (2) we first show that

$$
\left(\int_{E} f_{n}(t) \mu(d t)\right)
$$


is Cauchy uniformly with respect to $E \in \Sigma$. Let $p$ be a continuous seminorm, $\varepsilon>0$,

$$
\Phi(F)=\int_{F} g(t) \mu(d t)
$$

and $E_{n}=\left\{s \in S:\left|f(s)-f_{n}(s)\right| \geqq \varepsilon\right\}$. If $E \in \Sigma$ and $x^{*} \leqq p$, then $f$ is $x^{*} \mu$-integrable by the dominated convergence theorem for scalar measures and for each $n$

$$
\left|\int_{E}\left(f-f_{n}\right)(t) x^{*} \mu(d t)\right| \leqq \varepsilon\|\mu\|_{p}\left(E \backslash E_{n}\right)+2\|\Phi\|_{p}\left(E \cap E_{n}\right) .
$$

Thus

$$
\begin{aligned}
& p\left[\int_{E} f_{n}(t) \mu(d t)-\int_{E} f_{m}(t) \mu(d t)\right] \\
& \quad \leqq 2 \varepsilon\|\mu\|_{p}(S)+2\|\Phi\|_{p}\left(E_{n}\right)+2\|\Phi\|_{p}\left(E_{m}\right)
\end{aligned}
$$

for all $n$ and $m$. The sequence is Cauchy since $\lim _{n}\|\Phi\|_{p}\left(E_{n}\right)=0$.

The first assertion of (2) follows from this Cauchy condition and the dominated convergence theorem, and the second is true since

$$
\left(\int_{E} f_{n}(t) \mu(d t)\right)
$$

is uniformly Cauchy in $\mathscr{T}$ with respect to $E$ and converges weakly to $\int_{E} f(t) \mu(d t)$.

It follows that every bounded measurable function is $\mu$-integrable if $X$ is sequentially complete. The last two theorems of this section give characterizations of $\mu$-integrable functions when $X$ is sequentially complete.

Lemma 2.3. Let $\lambda$ be a complex valued measure on $(S, \Sigma)$ and $\left(f_{n}\right)$ a sequence of $\lambda$-integrable functions such that

(1) $\left(f_{n}\right)$ converges to $f$ pointwise on $S$, and

(2) $\left(\int_{E} f_{n}(t) \lambda(d t)\right)$ is Cauchy for each $E \in \Sigma$. Then $f$ is $\lambda$-integrable and $\int_{E} f(t) \lambda(d t)=\lim _{n} \int_{E} f_{n}(t) \lambda(d t)$ uniformly with respect to $E \in \Sigma$.

Proof. The proof follows the standard argument. For each $m$ define $\lambda_{m}$ on $\Sigma$ by $\lambda_{m}(E)=\int_{E} f_{m}(t) \lambda(d t)$. Each $\lambda_{m}$ is $v(\lambda)$-continuous and by $(2) \lim _{m} \lambda_{m}(E)$ exists for each $E \in \Sigma$. By the Vitali-Hahn-Saks Theorem $\lim _{v(\lambda, E) \rightarrow 0} \lambda_{m}(E)=0$ uniformly in $m$. Let $\varepsilon>0$ and

$$
E_{n}=\left\{s \in S:\left|f_{n}(s)-f(s)\right| \geqq \varepsilon\right\}
$$


for each $n$. By (1) $\lim _{n} E_{n}=\varnothing$, so $\sup _{n \geqq n_{0}} \sup _{m} v\left(\lambda_{m}, E_{n}\right)<\varepsilon$ for some $n_{0}$. Also, for $n \geqq n_{0}$,

$$
\begin{aligned}
& \int\left|f(t)-f_{n}(t)\right| v(\lambda, d t) \\
\leqq & \varepsilon v\left(\lambda, S \backslash E_{n}\right)+\lim \inf _{m} \int_{E_{n}}\left|f_{m}(t)-f_{n}(t)\right| v(\lambda, d t) \\
\leqq & \varepsilon[v(\lambda, S)+2] .
\end{aligned}
$$

This inequality establishes the lemma.

THEOREM 2.4. Suppose $X$ is sequentially complete and $f$ is a complex valued function on $S$. The following are equivalent:

(1) $f$ is $\mu$-integrable.

(2) There is a sequence $\left(f_{n}\right)$ of bounded measurable functions which converges pointwise to f and for which $\left(\int_{E} f_{n}(t) \mu(d t)\right)$ is Cauchy uniformly with respect to $E \in \Sigma$.

(3) There is a sequence $\left(f_{n}\right)$ of simple functions which converges pointwise to $f$ and for which $\left(\int_{E} f_{n}(t) \mu(d t)\right)$ is Cauchy for each $E \in \Sigma$.

Proof. For each $n$ let $E_{n}=\{s \in S:|f(s)| \leqq n\}$ and $f_{n}=f \chi_{E_{n}}$. If $f$ is $\mu$-integrable then $\left(f_{n}\right)$ satisfies condition (2) by Theorem 2.2. (2) clearly implies (3). To see that (3) implies integrability, let $E \in \Sigma$ and $x_{E}=\lim _{n} \int_{E} f_{n}(t) \mu(d t)$. For $x^{*} \in X^{*}$, an application of Lemma 2.3 with $\lambda=x^{*} \mu$ shows that

$$
x^{*}\left(x_{E}\right)=\lim _{n} \int_{E} f_{n}(t) x^{*} \mu(d t)=\int_{E} f(t) x^{*} \mu(d t) .
$$

Notice that if $f$ and $\left(f_{n}\right)$ satisfy condition (2) or (3) of Theorem 2.4, then, by Lemma 2.3,

$$
\int_{E} f(t) \mu(d t)=\lim _{n} \int_{E} f_{n}(t) \mu(d t)
$$

for each $E \in \Sigma$. Also, a reformation of (3) with the word Cauchy replaced by convergent is equivalent to (1) without sequential completeness.

We next consider the case in which $X$ is normed. Here we need only one semi-variation of $\mu$, that with $p(x)=\|x\|$. The dual of $X^{*}$ under its natural norm topology will be denoted by $X^{* *}$.

Definition 2.5. Suppose $X$ is a normed space. A function $f: S \rightarrow \not$ has a generalized integral with respect to $\mu$ if $f$ is $x^{*} \mu$ - 
integrable for each $x^{*} \in X^{*}$. If $f$ is such a function, then $\int_{E} f d \mu$ is the linear form on $X^{*}$ defined by $\left(\int_{E} f d \mu\right) x^{*}=\int_{E} f(t) x^{*} \mu(d t)$.

If $f$ is $\mu$-integrable, then $\int_{E} f d u$ is the image of $\int_{E} f(t) \mu(d t)$ under the natural map from $X$ into $X^{* *}$. $\int_{E} f d u$ is always in $X^{* *}$, since it is the pointwise limit of a sequence of the integrals of simple functions.

Theorem 2.6. Suppose $X$ is a Banach space and $f: S \rightarrow \phi$ has a generalized integral. The following are equivalent:

(1) $f$ is $\mu$-integrable.

(2) The set function $\Phi$ from $\Sigma$ into $X^{* *}$ defined by

$$
\Phi(E)=\int_{E} f d \mu
$$

is measure in the norm topology on $X^{* *}$.

(3) $\lim _{|| \mu||(E) \rightarrow 0}|| \int_{E} f d \mu \|=0$.

Proof. (1) implies (3) by Theorem 2.2. (2) is immediate from (3). For each $n$ let $F_{n}=\{s \in S:|f(s)| \leqq n\}$ and $f_{n}=f \chi_{E_{n}}$. For $n$ and $m$ positive integers and $E \in \Sigma$,

$$
\left\|\int_{E} f_{m}(t) \mu(d t)-\int_{E} f_{m}(t) \mu(d t)\right\| \leqq\|\Phi\|\left(S \backslash E_{n}\right)+\|\Phi\|\left(S \backslash E_{m}\right),
$$

so that $\left(f_{n}\right)$ satisfies condition (2) of Theorem 2.4 if $\Phi$ is a measure.

3. Below $S$ is a compact Hausdorff space, $\Sigma$ the Borel sets of $S, C(S)$ the Banach space (under supremum norm) of continuous complex valued functions on $S$ and $(X, \mathscr{T})$ a locally convex, Hausdorff linear topological space.

THEOREM 3.1. Let $A: C(S) \rightarrow X$ be a weakly compact linear operator. There is a measure $\mu: \Sigma \rightarrow X$ such that

(1) $\mu$ is regular,

(2) the closed absolutely convex hull of $\mu[\Sigma]$ is weakly compact,

(3) every bounded Borel function on $S$ is $\mu$-integrable,

(4) $A f=\int f(t) \mu(d t)$ for $f \in C(S)$, and

(5) $A^{*} x^{*}=x^{*} \mu$ for $x^{*} \in X^{*}$.

Conditions (1) and (4) define $\mu$ uniquely. $\|A\|=\|\mu\|(S)$ whenever $X$ is normed. If $\mu$ is a measure on $\Sigma$ which satisfies (1), (2), and 
(3), then (4) defined a weakly compact operator which satisfies (5). If $X$ is complete, then (2) and (3) follow from (1).

Proof. Since the dual of $C(S)$ may be identified with the bounded regular Borel measures on $\Sigma$, the equation

$$
g^{\wedge}(\lambda)=\int g(t) \lambda(d t)
$$

defines an element of $C(S)^{* *}$ for each bounded Borel function $g$. Since $A$ is weakly compact, $A^{* *}$, the algebraic adjoint of $A^{*}$, maps $C(S)^{* *}$ into $X$. For $E \in \Sigma$ let $\mu(E)=A^{* *}\left(\chi_{E}^{\wedge}\right)$. For each $x^{*} \in X^{*}$, $x^{*} \mu=A^{*} x^{*}$ is a regular measure, so $\mu$ is a regular measure. Since $A^{* *}$ maps the unit ball of $C(S)^{* *}$ into a weakly compact subset of $X$, condition (3) is satisfied. If $\sum_{i \leqq n} a_{i} \chi_{E_{i}}$ is a simple Borel function, then

$$
A^{* *}\left[\left(\sum_{i \leqq n} a_{i} \chi_{E_{i}}\right)^{\wedge}\right]=\sum_{i \leqq n} a_{i} A^{* *}\left(\chi_{E_{i}}^{\wedge}\right)=\int\left(\sum_{i \leqq n} a_{i} \chi_{E_{i}}\right)(t) \mu(d t) \text {. }
$$

Thus $x^{*}\left(A^{* *} g^{\wedge}\right)=\int g(t) x^{*} \mu(d t)$ holds for each bounded Borel function $g$ and $x^{*} \in X^{*}$. Finally, $\|A\|=\left\|A^{*}\right\|=\|\mu\|(E)$ if $X$ is normed.

Conversely, suppose $\mu$ satisfies (1), (2), (3). The operator $A$ defined by (4) is continuous since $p[A f] \leqq\|f\|\|\mu\|_{p}(S)$ for each continuous semi-norm $p$ on $X$. Also, by the regularity of $\mu, A^{*} x^{*}=x^{*} \mu$ for $x^{*} \in X^{*}$. Let $U$ be the polar of the closed, absolutely convex hull of $\nu[\Sigma] . \quad U$ is a neighborhood of zero in the Mackey topology on $X^{*}$, and, for $x^{*} \in U,\left\|A^{*} x^{*}\right\| \leqq 4$. Thus $A^{*}$ is continuous with the Mackey topology on $X^{*}$ and the norm topology on $C(S)^{*}$-this implies that $A$ is weakly compact.

If $X$ is complete and $\mu$ is regular, then $A f=\int f(t) \mu(d t)$ defines a continuous linear operator for $C(S)$ into $X$ such that $A^{*} x^{*}=x^{*} \mu$ for $x^{*} \in X^{*}$. To see that (2) holds, it is sufficient to show that $A$ is weakly compact-equivalently, that $A^{*}$ maps equicontinuous sets into weakly relatively compact sets. Let $V$ be an open neighborhood of zero in $X$ generated by a semi-norm $p$. For $x^{*} \in V^{\circ}$ and $E \in \Sigma$,

$$
\left|x^{*} \mu(E)\right| \leqq\|\mu\|_{p}(E),
$$

so the countable additivity of $A^{*}\left[V^{\circ}\right]$ is uniform. This together with norm boundedness implies that $A^{*}\left[V^{\circ}\right]$ is relatively weakly compact.

In [1] Bartle, Dunford and Schwartz have given a similar integral representation for weakly compact operators from $C(S)$ into a Banach space. Grothendieck [5] has noted that there is a one-to-one correspondence between the weakly compact operators from $C(S)$ into a complete, locally convex, Hausdorff space $X$ and the $X$-valued measures on the Baire sets of $S$, although he did not give an integral repre- 
sentation of such operators.

Let $T$ be a locally compact Hausdorff space. $C_{0}(T)\left[C_{c}(T)\right]$ is the Banach space under supremum norm of complex valued functions on $T$ which vanish at infinity [have compact support]. $C(T)_{\beta}$ is the space of bounded, continuous, complex valued functions on $T$ topologized by the semi-norms $p_{\varphi}(f)=\sup _{t \in T}|\varphi(t) f(t)|$, where $\varphi \in C_{0}(T)$. A weakly compact operator $A: C_{0}(T) \rightarrow X$ can be represented by integration with respect to an $X$-valued measure since $A$ can be extended to a weakly compact operator on the space of continuous functions over the one point compactification of $T$. Weakly compact operators on $C_{c}(T)$ have such a representation since they can be extended to $C_{0}(T)$. The bounded sets of $C(T)_{\beta}$ are precisely the uniformly bounded sets and each element of $C(T)_{\beta}^{*}$ can be identified with a bounded regular Borel measure on $T$ [2], so the proof of Theorem 3.1 generalizes immediately for $C(S)_{\beta}$.

COROLLARY 3.2. Let $A$ be a weakly compact operator from one of $C(S), C_{0}(T), C_{c}(T)$ or $C(T)_{\beta}$ into $X$. A maps weakly Cauchy sequences into convergent sequences.

Proof. If $\left(f_{n}\right)$ is weakly Cauchy in any of the above spaces, then $\left(f_{n}\right)$ is uniformly bounded and converges pointwise to a bounded Borel function $f$. Let $\mu$ be the measure determining $A$. $f$ is $\mu$-integrable and, by Theorem 2.1, $\int f(t) \mu(d t)=\lim _{n} A f_{n}$.

This is proven in [5] for $C(S)$ under the assumption that $X$ is complete.

\section{BIBLIOGRAPHY}

1. R. G. Bartle, N. Dunford, and J. Schwartz, Weak compactness and vector measures, Canad. J. Math. 7 (1955), 289-305.

2. R. C. Buck, Bounded continuous functions on a locally compact space, Michigan Math. J. 5 (1958), 95-104.

3. N. Dunford, and J. Schwartz, Linear operators, Part I, Interscience, New York, 1958.

4. J. G. de Lamadrid, Measures and tensors, Trans. Amer. Math. Soc. 114 (1965), 98-121.

5. A. Grothendieck, Sur les applications linéaries faiblement compactes d'espaces du type $C(K)$, Canad. J. Math. 5 (1953), 129-173.

6. B. J. Pettis, On integration in vector spaces, Trans. Amer. Math. Soc. 44 (1938), 277-304.

Received February 10, 1969.

Louisiana State UNIVERSity

BATON ROUGE, LOUISIANA 



\section{PACIFIC JOURNAL OF MATHEMATICS}

\section{EDITORS}

H. SAMELSON

Stanford University

Stanford, California 94305

\section{RichaRd PIERCe}

University of Washington

Seattle, Washington 98105
J. DUGUNDJI

Department of Mathematics

University of Southern California

Los Angeles, California 90007

RICHARD ARENS

University of California

Los Angeles, California 90024

\section{ASSOCIATE EDITORS}

E. F. BECKENBACH

B. H. NeUManN

F. WOLF

K. YosHIDA

\section{SUPPORTING INSTITUTIONS}

UNIVERSITY OF BRITISH COLUMBIA CALIFORNIA INSTITUTE OF TECHNOLOGY UNIVERSITY OF CALIFORNIA MONTANA STATE UNIVERSITY

UNIVERSITY OF NEVADA

NEW MEXICO STATE UNIVERSITY

OREGON STATE UNIVERSITY

UNIVERSITY OF OREGON

OSAKA UNIVERSITY

UNIVERSITY OF SOUTHERN CALIFORNIA
STANFORD UNIVERSITY

UNIVERSITY OF TOKYO

UNIVERSITY OF UTAH

WASHINGTON STATE UNIVERSITY

UNIVERSITY OF WASHINGTON

${ }^{*} \quad{ }^{*} \quad{ }^{*}$
AMERICAN MATHEMATICAL SOCIETY
CHEVRON RESEARCH CORPORATION
TRW SYSTEMS
NAVAL WEAPONS CENTER




\section{Pacific Journal of Mathematics}

\section{Vol. 33, No. $1 \quad$ March, 1970}

Mir Maswood Ali, On some extremal simplexes ................... 1

Silvio Aurora, On normed rings with monotone multiplication........... 15

Silvio Aurora, Normed fields which extend normed rings of integers....... 21

John Kelly Beem, Indefinite Minkowski spaces..................... 29

T. F. Bridgland, Trajectory integrals of set valued functions ........... 43

Robert Jay Buck, A generalized Hausdorff dimension for functions and sets ......................................... 69

Vlastimil B. Dlab, A characterization of perfect rings . . . . . . . . . . . . 79

Edward Richard Fadell, Some examples in fixed point theory ............ 89

Michael Benton Freeman, Tangential Cauchy-Riemann equations and uniform approximation ............................. 101

Barry J. Gardner, Torsion classes and pure subgroups ................ 109

Vinod B. Goyal, Bounds for the solution of a certain class of nonlinear

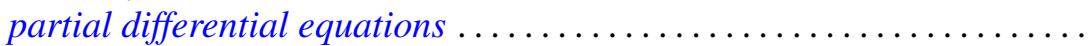

Fu Cheng Hsiang, On C, 1 summability factors of Fourier series at a given

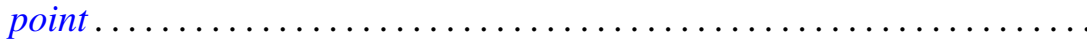

Lawrence Stanislaus Husch, Jr., Homotopy groups of PL-embedding

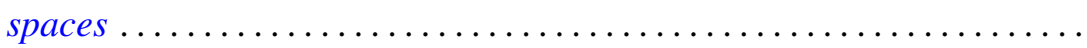

Daniel Ralph Lewis, Integration with respect to vector measures..........

Marion-Josephine Lim, $\mathscr{L}-2$ subspaces of Grassmann product spaces

Stephen J. Pierce, Orthogonal groups of positive definite multilinear functionals

W. J. Pugh and S. M. Shah, On the growth of entire functions of bounded index.

Siddani Bhaskara Rao and Ayyagari Ramachandra Rao, Existence of triconnected graphs with prescribed degrees . . .

Ralph Tyrrell Rockafellar, On the maximal monotonicity of subdifferential

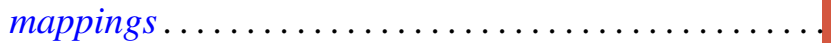

R. Shantaram, Convergence of a sequence of transformations of distribution functions. II ...............................

Julianne Souchek, Rings of analytic functions..............

Ted Joe Suffridge, The principle of subordination applied to functions of several variables...

Wei-lung Ting, On secondary characteristic classes in cobordism

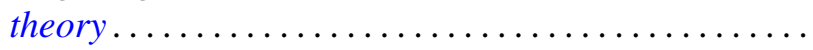

Pak-Ken Wong, Continuous complementors on $B^{*}$-algebras ...

Miyuki Yamada, On a regular semigroup in which the idempotents form a band. 